TecnoLógicas

ISSN-p 0123-7799

ISSN-e 2256-5337

Vol. 23, No. 49, pp. 201-221

Sep-dic de 2020

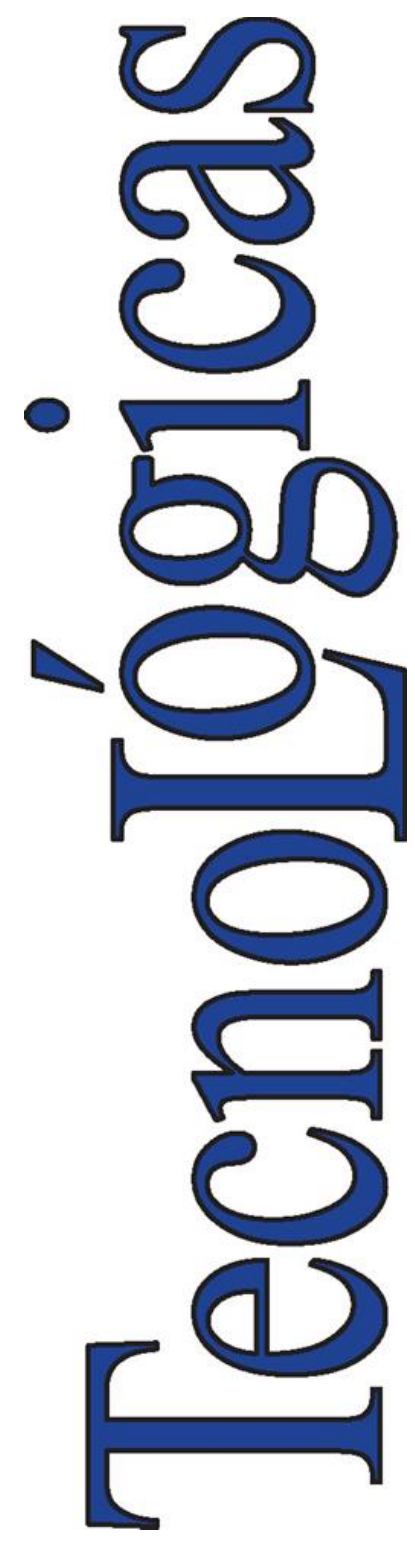

(C) Instituto Tecnológico Metropolitano Este trabajo está licenciado bajo una Licencia Internacional Creative Commons Atribución (CC BY-NC-SA)
Artículo de investigación/Research Article

\section{Herramienta basada en agentes para la valoración del impacto de intervenciones no farmacéuticas contra la COVID-19}

\section{An agent-based tool for impact assessment of non-pharmaceutical interventions against COVID-19}

\author{
Lindsay Álvarez Pomar (D) 1 \\ Sergio Rojas Galeano (D)
}

Recibido: 4 de junio de 2020

Aceptado: 25 de agosto de 2020

Cómo citar / How to cite

L. Álvarez-Pomar, S. Rojas-Galeano, "Herramienta basada en agentes para la valoración del impacto de intervenciones no farmacéuticas contra la COVID-19", TecnoLógicas, vol. 23, no. 49, pp. 201-221, 2020. https://doi.org/10.22430/22565337.1685

1 PhD. en Ingeniería, Facultad de Ingeniería, Universidad Distrital Francisco José de Caldas, Bogotá-Colombia, lalvarez@udistrital.edu.co

2 PhD. en Ciencia Computacional, Facultad de Ingeniería, Universidad Distrital Francisco José de Caldas, Bogotá-Colombia, srojas@udistrital.edu.co 


\title{
Resumen
}

Las intervenciones no farmacéuticas (NPI) son actualmente el único mecanismo que los gobiernos pueden usar para mitigar el impacto de la epidemia de COVID-19. De manera similar a la propagación real de la enfermedad, la dinámica de los patrones de contención que surgen de la aplicación de los NPI es compleja y depende de las interacciones entre las personas dentro de una región específica, así como de otros factores estocásticos asociados a condiciones demográficas, geográficas, políticas y económicas. Los modelos basados en agentes simulan reglas microscópicas de interacciones simultáneas de múltiples individuos dentro de una población en un intento de reproducir la dinámica compleja del efecto de las medidas de contención. De esta manera, es posible diseñar comportamientos individuales junto con escenarios de NPI, midiendo cómo se ve afectada la dinámica de la simulación y, por lo tanto, brindando información útil para realizar una evaluación rápida del potencial de las intervenciones combinadas, en las diferentes etapas de la epidemia. En este artículo describimos un modelo y una herramienta para experimentar con este tipo de análisis, considerando una serie de NPI ampliamente utilizadas, tales como distanciamiento físico, aislamiento de casos, cuarentena domiciliaria, encierro total, pruebas centinela, uso de tapabocas y una novedosa aplicación "zonal", que permite aplicar estas intervenciones gradualmente a localidades o zonas separadas. La elección de las intervenciones o combinaciones más adecuadas para un territorio particular, dependerá en última instancia de las condiciones socioeconómicas y de salubridad, así como de una validación a gran escala de la viabilidad de los escenarios identificados preliminarmente mediante la herramienta.

\section{Palabras clave}

Contención de la epidemia COVID-19, evaluación de intervenciones no farmacéuticas, simulación y herramientas orientadas a agentes.

\begin{abstract}
Non-Pharmaceutical Interventions (NPI) are currently the only mechanism governments can use to mitigate the impact of the COVID-19 epidemic. Similarly, to the actual spread of the disease, the dynamics of the contention patterns emerging from the application of NPIs are complex and depend on interactions between people within a specific region as well as other stochastic factors associated to demographic, geographic, political and economical conditions. Agent-based models simulate microscopic rules of simultaneous interactions of multiple agents within a population in an attempt to reproduce the complex dynamics of the effect of the contention measures. In this way it is possible to design individual behaviors along with NPI scenarios, measuring how the simulation dynamics is affected and therefore, yielding rapid insights to perform a broad assessment of the potential of composite interventions at different stages of the epidemic. In this paper we describe a model and a tool to experiment with this kind of analysis, considering a number of widely-applied NPIs such as social distancing, case isolation, home quarantine, total lockdown, sentinel testing, mask wearing and a novel "zonal" enforcement requiring these interventions to be applied gradually to separated districts (zones). The choice of the most adequate interventions, or mixture of interventions, ultimately will depend on the socio-economic and health conditions of a particular territory and on further large-scale simulation and feasibility estimation of those scenarios yielding a potential mitigation impact, using the insights discovered with the simulation tool.
\end{abstract}

\section{Keywords}

COVID-19 epidemic contention, Non-Pharmaceutical Interventions assessment, Agentbased simulation and tools. 


\section{INTRODUCCIÓN}

En vista de la ausencia de medicamentos o vacunas aprobados para COVID-19 (hasta el día de hoy), las intervenciones no farmacéuticas (NPI, acrónimo de Non-Pharmaceutical Interventions) son los mecanismos que las secretarías de salud pública de todo el mundo están utilizando con la esperanza de mitigar el impacto de esta pandemia [1], [2]. De manera similar a la propagación real de la enfermedad, la dinámica de los patrones de contención obtenidos como resultado de la aplicación de NPI es compleja y puede depender no solo de las interacciones (contactos) entre las personas según su comportamiento individual, sino de muchos otros factores estocásticos, asociados a condiciones demográficas, geográficas, políticas y económicas.

Una forma de obtener información sobre la progresión de las epidemias para permitir que los investigadores y los gobernantes tomen medidas para controlar su propagación, son los modelos de simulación. En este sentido, existen dos enfoques principales. El enfoque más conocido utiliza modelos macroscópicos que representan dinámicas a nivel de población con análisis de causalidad o mediante el modelado de eventos discretos que afectan el estado del sistema (Dinámica de Sistemas, Simulación de Eventos Discretos). Este tipo de modelos están destinados a describir la dinámica de infección promedio haciendo uso de ecuaciones diferenciales y se han utilizado en el pasado para estudiar la propagación de las pandemias de influenza [3] y más recientemente para COVID-19 [2].

Estos se basan en el modelo epidémico clásico de compartimentos llamado SIR (Susceptible, Infeccioso, Recuperado) o variaciones del mismo, en los que la población entera se divide en dichos compartimentos y la dinámica de la epidemia se explica por la evolución de la enfermedad en cada compartimento en lugar de a nivel individual. Los parámetros del modelo, como las tasas de transición entre compartimentos, se calibran con los datos disponibles, mientras que otros parámetros como el número de reproducción o la configuración de las NPI, se asumen para simular escenarios hipotéticos, ver por ejemplo: [4], [5], [6], [7], [8], [2], [1].

El otro enfoque recurre al modelado microscópico de interacciones simuladas a nivel individual, también conocido como microsimulación; aquí los fenómenos globales se observan como una propiedad emergente de la dinámica colectiva.

Comúnmente conocidos como modelos basados en individuos o IBM, este enfoque asume una estructura de red inherente dentro de la población, produciendo contagios dependientes de la interacción (o enlaces) entre individuos, mientras que la aparición de síntomas y el progreso de la enfermedad se modelan con distribuciones de probabilidad condicionadas por las estructuras de edad o demográficas. Recientemente se han propuesto varios modelos de este tipo para estudiar la propagación y la contención de COVID-19, ver por ejemplo [9], [10], [11].

Este último enfoque de microsimulación también se conoce como modelado basado en agentes o ABM [12]. Algunas ventajas del enfoque ABM para estudiar la epidemia incluyen su capacidad para modelar decisiones deterministas y no deterministas tomadas por los individuos afectados por interacciones con otros agentes dentro de una vecindad espacial, lo que permite una simulación más intuitiva y cercana al sistema real. De esta manera, la incertidumbre o la varianza surge inherentemente en un ABM, lo que permite que el modelo obtenga emergencias no deterministas sin variación de parámetros de entrada [13].

La aleatoriedad de los patrones espaciales seguidos por la libre circulación de agentes, permite una representación más cercana a la heterogeneidad 
epidemiológicamente relevante de la población. Además, la posibilidad de establecer rasgos individuales particulares, así como el comportamiento y la progresión de la enfermedad por individuo, son características útiles para estudiar cómo los efectos no deterministas emergen de la aplicación de una combinación de diversas NPIs.

En este artículo proponemos un modelo $\mathrm{ABM}$ que incorpora una serie de NPIs, incluyendo distanciamiento físico, aislamiento de casos, cuarentena domiciliaria, encierro total, pruebas centinela, mascarillas (tapabocas) de protección y una novedosa propuesta de restricción "zonal", donde estas intervenciones se pueden aplicar a distritos o zonas separadas para una ciudad hipotética. El efecto de estas estrategias es medido en términos de morbilidad, mortalidad, tasas de letalidad, incluidas tasa de fatalidad según infecciones (IFR, por sus siglas en inglés) y tasa de fatalidad según casos confirmados (CFR, por sus siglas en inglés), tiempo de duplicación, número de reproducción y gráficos de recuperación de la infección y muerte durante la línea de tiempo de la simulación. El principal aporte de la herramienta es permitir que los modeladores vean cuáles NPIs o combinación de NPIs pueden ayudar a aplanar o aplastar la curva de propagación de la enfermedad (mitigar o suprimir, respectivamente), así como evaluar los efectos sobre los indicadores de epidemias correspondientes, a través de una representación más detallada de condiciones simultáneas que puede tener un agente. La descripción de las NPI contempladas, las características del modelo y la herramienta desarrollada se dan en las siguientes secciones, junto con los resultados de escenarios de simulación hipotéticos.

\section{INTERVENCIONES NO FARMACÉUTICAS}

Las NPI son políticas de salud destinadas a mitigar los efectos de la propagación de un nuevo virus cuando las vacunas o medicamentos aún no están disponibles. Consisten en acciones recomendadas al público o, en algunos casos, impuestas por el gobierno que afectan sus hábitos de vida cotidianos.

Durante la pandemia de COVID-19, muchos de estos planes de acción se han diseñado y desplegado a escala mundial, con diferentes resultados según la región geográfica. Con base en la literatura y en las políticas de salud pública que la mayoría de países han adoptado para contener la pandemia, a continuación, describimos las NPI que están incorporadas actualmente en nuestro modelo; adicionalmente proponemos una alternativa que permite la combinación de varias NPI con restricciones de movilidad zonal.

\subsection{Distanciamiento Físico (Social Distancing, SD)}

Esta intervención consiste en mantener una distancia física mínima entre las personas para reducir el número de interacciones cercanas entre individuos infectados y sanos y, por lo tanto, reducir la velocidad de transmisión y eventuales muertes. En el modelo, esta intervención se denomina Distancia Social SD, se puede activar o desactivar, y la cantidad real de personas dispuestas a cumplir con la intervención se puede definir con un parámetro de porcentaje entre 5 y $100 \%$.

\subsection{Aislamiento de Casos (Case Isolation, CI)}

Esta intervención consiste en confinar a los casos confirmados en una instalación aislada (hogar) o a pacientes suficientemente sintomáticos para autoconfinarse, hasta que se recuperen de la 
enfermedad. En el modelo, esta intervención se puede activar o desactivar.

\subsection{Cuarentena Quarantine, HQ)}

Domiciliaria (Home

Esta intervención consiste en el confinamiento de familiares o compañeros de residencia que viven en el mismo hogar de casos aislados, durante su recuperación.

Se supone que las personas sanas en cuarentena en el hogar deben observar estrictos protocolos de seguridad para evitar el contagio del caso aislado. En el modelo, esta intervención se puede activar o desactivar.

\subsection{Encierro Total (Total Lockdown, TL)}

Esta intervención impone una política de quedarse en casa, con el correspondiente cierre parcial de la economía. Solo algunas personas están autorizadas a desplazarse para satisfacer las necesidades básicas o para apoyar los servicios esenciales. En el modelo, esta intervención se puede activar o desactivar con un porcentaje de permisos entre 5 y 95 $\%$.

\subsection{Pruebas Centinela (Sentinel Testing, ST)}

Esta intervención consiste en ejecutar campañas de salud que realicen pruebas masivas en ubicaciones aleatorias para detectar casos no aislados, sintomáticos o asintomáticos, no confirmados previamente. Las personas cuyo resultado dé positivo en la prueba, se aíslan inmediatamente. En el modelo, esta intervención puede activarse o desactivarse, con la opción de desplegar las campañas a lo largo de la ciudad o restringida por zonas. El inventario de pruebas disponibles es un parámetro del modelo definido como una fracción de la población entre 10 y $100 \%$. En caso de que las campañas agoten las pruebas, es posible reponer el inventario con un nuevo suministro de la misma cantidad.

\subsection{Restricción Zonal (Zonal Enforcement, ZE)}

Esta intervención supone que la ciudad hipotética puede dividirse en diferentes distritos (o zonas), y luego implementa la restricción del movimiento de los residentes solo dentro de sus respectivas zonas. Las demás NPIs se pueden aplicar localizados en cada zona. Nuestro modelo también incluye una novedosa NPI que consiste en aplicar un encierro total a toda la ciudad, con la opción de levantar gradualmente el toque de queda por zona.

En el simulador, esta intervención se puede activar o desactivar, además de ofrecer un comando para desbloquear una zona a la vez.

\subsection{Mascarillas de Protección (Mask Protection, MP)}

Esta intervención consiste en el uso de máscaras faciales como equipo de protección personal para evitar la propagación o la respiración de partículas de virus en el aire. En el modelo, esta intervención se simula en realidad como un hábito poblacional controlado por una combinación de dos parámetros: portadores (personas infectadas) que usan mascarillas o no, y susceptibles (personas sanas) que usan mascarillas o no. Una combinación particular tendrá incidencia en la posibilidad de contagio cuando dos personas se encuentren: $3 \%$ si ambos usan máscaras, $8 \%$ si el portador usa máscaras pero los susceptibles no, $50 \%$ si $\operatorname{los}$ susceptibles usan máscaras pero el portador no y $90 \quad \%$ si ninguno usa mascarillas. 


\section{MODELO BASADO EN AGENTES}

\subsection{Modelo epidemiológico}

Nuestro modelo basado en agentes utiliza el modelo de epidemias compartimental SIRE (SusceptibleInfectious-Recovered-Extinct), pero considerando el compartimento $I$ como un estado extendido [14], que consta de una serie de condiciones que creemos explican mejor la dinámica de la infección. Estas condiciones son las siguientes: Confirmed o no (lo que indica que el paciente permanecerá aislado), Risky o no (lo que significa predisposición a desarrollar una enfermedad grave o crítica), Asymptomatic o no (lo que significa que el paciente no es consciente de ser portador del virus), Severe o no (lo que significa que debe ser hospitalizado para evitar la muerte) y Deadly o no (lo que indica que el paciente requiere asistencia de la Unidad de Cuidados Intensivos - UCI).

La representación esquemática del modelo extendido resultante, que llamamos $S i ̂ R E_{C A R D S}$, se muestra en la Fig. 1. Nótese que no todas estas condiciones son mutuamente excluyentes, ya que representan etapas acumuladas del estado î, que evolucionan de la manera que se explica más adelante.

En este sentido diseñamos tres tipos de agentes: un agente saludable asociado con los estados $S$ y $R$ (este último etiquetado con una condición de inmunidad), un agente enfermo asociado con el estado î extendido (etiquetado con las condiciones $C A R D S)$ y un agente extinto asociado con el $E$ estado. A diferencia de la simulación de dinámica de población, en lugar de usar tasas de transición entre estados, nuestro modelo usa eventos de transición.
Un evento de transición es un conjunto de reglas de decisión evaluadas en las condiciones de cada estado. En este sentido, nuestra propuesta difiere de otros enfoques ABM similares, donde estas condiciones se consideran compartimentos adicionales de modelos SIR modificados, por ejemplo, SIDHARTE [7], REINA [10], SEAIRHD [15] o INFEKTA [11].

La propuesta de considerar un estado extendido definido por las condiciones CARDS mencionadas anteriormente, permite obtener una representación más detallada de los agentes, potenciando la posibilidad de analizar escenarios donde la evolución del fenómeno ocurra en subpoblaciones afectadas simultáneamente por varias condiciones. Por ejemplo, sería posible hacer seguimiento a agentes $o$ individuos asintomáticos que a la vez sean población de alto riesgo. Lo anterior no sería factible haciendo uso de modelos con compartimentos excluyentes para cada subgrupo.

Se consideraron los siguientes eventos de transición:

$S \rightarrow I$ (infección). Un individuo sano puede infectarse (con una probabilidad de contagio $p$-contagion) si ocurre contacto con otro individuo infeccioso. Cuando ocurre la transmisión, el nuevo caso se caracteriza inicialmente como no confirmado, no testado, no grave, no mortal, no hospitalizado y no ingresado en la UCI.

Además, se asigna un período de recuperación en días como una variante aleatoria con una distribución Gaussiana centrada en el parámetro de duración promedio del modelo, avg-duration. 


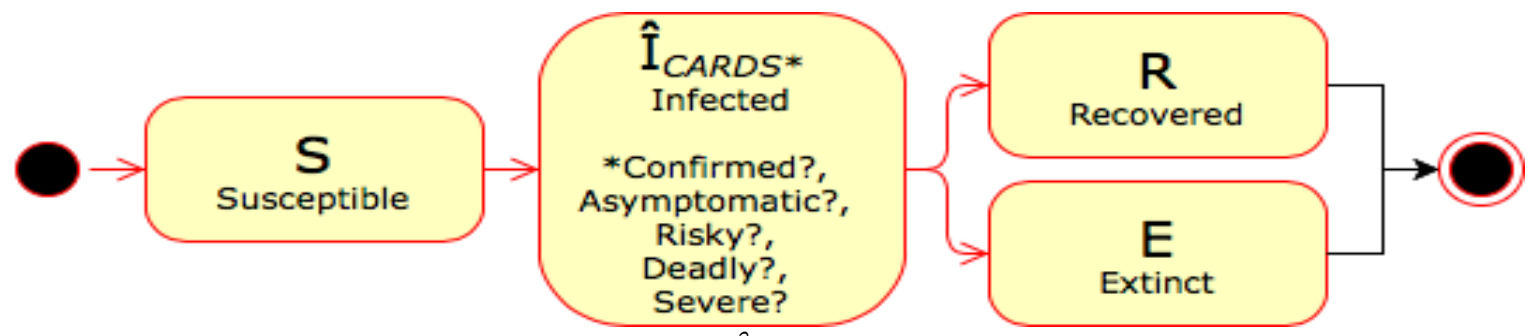

Fig. 1. Esquema del modelo SîRE $C A R D S$. Fuente: elaboración propia.

$\hat{I}+$ Confirmed. Esta condición caracteriza a un agente como un caso positivo. Esto puede suceder en cualquiera de los siguientes momentos: cuando al paciente se le realiza la prueba del virus y los resultados de la prueba son positivos; si en el momento de ser ingresado al hospital, o la cama de la UCI o cuando fallece, el agente no había sido diagnosticado previamente como positivo; o cuando el paciente se siente lo suficientemente sintomático para auto-aislarse. Por otro lado, si el NPI de aislamiento de casos (ver arriba) se levanta después de haber sido aplicado durante el curso de la simulación, entonces la condición de confirmado de los agentes que no han resultado positivos, se revierte (es decir, la de aquellos que se auto-aíslan).

La distinción entre casos confirmados y no confirmados se usa para identificar individuos que necesitan permanecer aislados para mitigar su capacidad de propagar el virus. Además, esta distinción también es útil para examinar la discrepancia entre las tasas de mortalidad confirmadas "oficiales" y la tasa de letalidad real (que se obtiene respecto a todos los infectados, no solo confirmados).

$\hat{l}+$ Asymptomatic. Esta condición caracteriza a un agente como que no muestra síntomas de la enfermedad. La condición se activa al adquirir la infección, con una probabilidad definida como un parámetro modelo (\%-asymptomatic). En la vista de la simulación, esta condición refleja el color del agente (amarillo para asintomático, rojo para sintomático).

$\hat{\imath}+$ Risky. Esta condición caracteriza a un agente como parte de un grupo de población de alto riesgo. Actualmente, el modelo no considera la estratificación del riesgo de las comunidades ni la estructura de edad de la población. Por lo tanto, todos estos factores de riesgo (obesidad, diabetes, enfermedad cardiovascular o edad media a avanzada) están abarcados en esta condición única que se activa al adquirir la infección, con una probabilidad definida como un parámetro modelo (\%-high risk).

$i+$ Severity. Esta condición indica la progresión de la enfermedad a una condición de gravedad, que a su vez representa una mayor amenaza de muerte, por ser requisito previo para pasar a la condición crítica. Este evento ocurre de acuerdo con las siguientes probabilidades [10]:

$P($ Severe $\mid$ Symptomatic, Risky $)=0.25$,

$P($ Severe $\mid$ Symptomatic, no Risky $)=0.05$, $P($ Severe $\mid$ Asymptomatic $)=0.0$.

El día exacto en que se desencadena el evento de gravedad, se modela con una distribución uniforme durante el período de la enfermedad: $P($ day $)=\operatorname{CDF}-\operatorname{Uniform}(x<$ day), y la ocurrencia de la gravedad en un día arbitrario, así:

$P($ Severe $\mid$ day $)=P($ day $) P($ Severe | Conditions $)$.

$\hat{I}+$ Deadliness. Esta condición indica la progresión de la enfermedad a una condición crítica, que a su vez representa una amenaza aún mayor de muerte, como se verá en la transición $\hat{I} \rightarrow E$. Este evento ocurre de acuerdo con las siguientes probabilidades [10]: 
Herramienta basada en agentes para la valoración del impacto de intervenciones no farmacéuticas contra la COVID-19

$P($ Deadly $\mid$ Severe $)=0.25$,

$P($ Deadly $\mid$ no Severe $)=0.05$,

Del mismo modo, modelamos el día en que se desencadena el evento de condición crítica distribuido uniformemente dentro del período de la enfermedad:

$$
P(d a y)=\text { CDF-Uniform }(x<d a y) \text {. }
$$

Entonces, la ocurrencia de condición crítica en un día dado es:

$P($ Deadly $\mid d a y)=P($ day $) P($ Deadly $\mid$ Conditions $)$.

$\hat{\imath}+$ Hospitalised. Este evento indica que se está cuidando adecuadamente la condición de gravedad; por lo tanto, reduce la posibilidad de muerte, como se explica en la transición $\hat{I} \rightarrow E$. La ocurrencia de este evento depende de la disponibilidad de camas de hospital (definidas por el parámetro hospital-beds). Básicamente, tan pronto como se vacía una cama, se le asigna a un agente en estado de gravedad que se encuentre en espera (si lo hay), hasta que se alcanza la disponibilidad. Cuando un paciente ingresa, su estado cambia a hospitalizado, testado y confirmado (los dos últimos en caso de que no haya sido diagnosticado previamente).

La ocupación y la demanda de estas camas se actualizan diariamente. Nótese que las camas se liberan cuando los agentes infecciosos se recuperan $o$ se trasladan al tratamiento de la UCI o cuando fallecen.

$\hat{\imath}+$ ICU-admitted. Similar al evento anterior, este se refiere al tratamiento adecuado para la condición crítica que se está dando, por lo tanto, reduce la posibilidad de muerte, como se explica en la transición $\hat{I} \rightarrow E$. La asignación, el alta y la disponibilidad de camas en la UCI se realiza de forma análoga a las camas de los hospitales, a diario, pero considerando a los pacientes con una condición crítica. En este caso, las camas de la UCI se liberan cuando los agentes se recuperan o mueren. $i \rightarrow E$ (extinción). Un individuo infectado puede fallecer con una probabilidad que depende de sus condiciones, de la siguiente forma [10]:

$\mathrm{P}($ Extinct $\mid$ Deadly, no ICU-admitted $)=1$, $P($ Extinct $\mid$ Deadly, ICU-admitted $)=0.5$, $P($ Extinct $\mid$ Severe, no Hospitalised $)=0.2$, $P($ Extinct $\mid$ Severe, Hospitalised $)=0.05$,

$P($ Extinct $\mid$ Severe, Symptomatic $)=0.005$.

Dado que la muerte puede ocurrir en cualquier día durante el período de la enfermedad, modelamos la probabilidad del día de la muerte como una distribución triangular con un pico en la mitad del período de recuperación particular de dicho individuo (half-days). En otras palabras, suponemos:

$P($ day $)=$ CDF-Triangular $(x<$ day $\mid$ half-days $)$,

donde CDF corresponde a la función de distribución acumulativa. Como resultado, calculamos la probabilidad real de muerte en un día dado, de la siguiente manera:

$P($ Extinct $\mid d a y)=P($ day $) P($ Extinct $\mid$ Conditions $)$.

En la vista de la simulación, los agentes en este estado aparecen como una "X" de color negro.

$\hat{I} \rightarrow R$ (recuperación). Una persona infectada se cura cuando logra llegar al final de su período de recuperación.

$\mathrm{Si}$ esto sucede, nuestro modelo supone que el individuo adquiere inmunidad al virus y no puede volver a infectarse. En la vista de la simulación, los agentes en este estado aparecen de color blanco.

\subsection{Diseño de los agentes y de los comportamientos}

Los agentes representan a personas individuales que residen en una ciudad hipotética, cuyos atributos incluyen: ubicación espacial, residencia familiar $\mathrm{y}$ 
zona o distrito de la ciudad. El modelo rastrea una ruta de enfermedad individual para cada agente, desde susceptible hasta recuperación o muerte, de acuerdo con el modelo epidémico SîRE $E_{C A R D S}$ descrito anteriormente. A cada agente se le asigna una rutina diaria que consiste en salir al aire libre y regresar a casa con un rango de cobertura de distancia de viaje elegido al azar entre $\{25,50,100,200\}$ pasos unitarios; el camino real que sigue el agente varía ligeramente debido a fluctuaciones aleatorias en su orientación.

Además, la longitud de cada paso se puede establecer como un parámetro global entre 0.1 y 1 unidades. Del mismo modo, la duración del día se puede definir con un número determinado de tictacs en el rango entre 600 y 2400.

Las personas interactúan por contactos aleatorios que hicieron dentro de su hogar o durante sus rutinas diarias al aire libre.

La transmisión del virus ocurre debido a la proximidad de un individuo infeccioso con otros individuos susceptibles a su alrededor dentro de un radio espacial de 0.5 unidades. La posibilidad de contagio depende de la NPI de Mascarillas de Protección que se aplique, como se explicó anteriormente. Además, nuestro modelo no considera los períodos de incubación; se asume que la infecciosidad comienza tan pronto como ocurre el contagio, sin considerar diferencias entre la infectividad de portadores sintomáticos y asintomáticos, o infecciosidad variable entre individuos. Al recuperarse de la enfermedad, se asume que los individuos adquieren inmunidad al virus para que no puedan volver a infectarse. En este modelo, todas las muertes se consideran causadas por COVID-19. No se tienen en cuenta los nacimientos durante la línea de tiempo de la simulación.

En la Fig. 2 se muestra un esquema de alto nivel del flujo de eventos que se aplican en cada iteración de la simulación.

Seguidamente, describiremos cada uno de ellos.

lifestyle: simula la rutina diaria de los agentes, que actualmente incluye mover al agente hacia su destino actual, o regresarlo a casa si ha alcanzado su límite de cobertura. En todo caso, los pacientes confirmados no pueden moverse.

Si la NPI de restricción zonal está activada, el movimiento será restringido solo dentro de la periferia de la zona donde reside el agente. En la vista de simulación, los residentes de diferentes zonas se identifican con diferentes formas y colores (este último es del mismo color que el suelo de la zona).

epidemic: aquí se simula la propagación de la epidemia, con transmisiones que se producen en proporción a la proximidad entre infecciosos y susceptibles y la probabilidad de contagio. Si ocurre una transmisión, el nuevo caso se caracteriza inicialmente como no confirmado, no testado, no grave y no mortal. La condición de asintomático y/o riesgoso de un nuevo caso se define de acuerdo con las probabilidades correspondientes establecidas como parámetros del modelo.

Por último, el período de recuperación del agente en días, se asigna como una variante aleatoria con una distribución Gaussiana centrada en el parámetro del modelo de duración promedio (avgduration). 


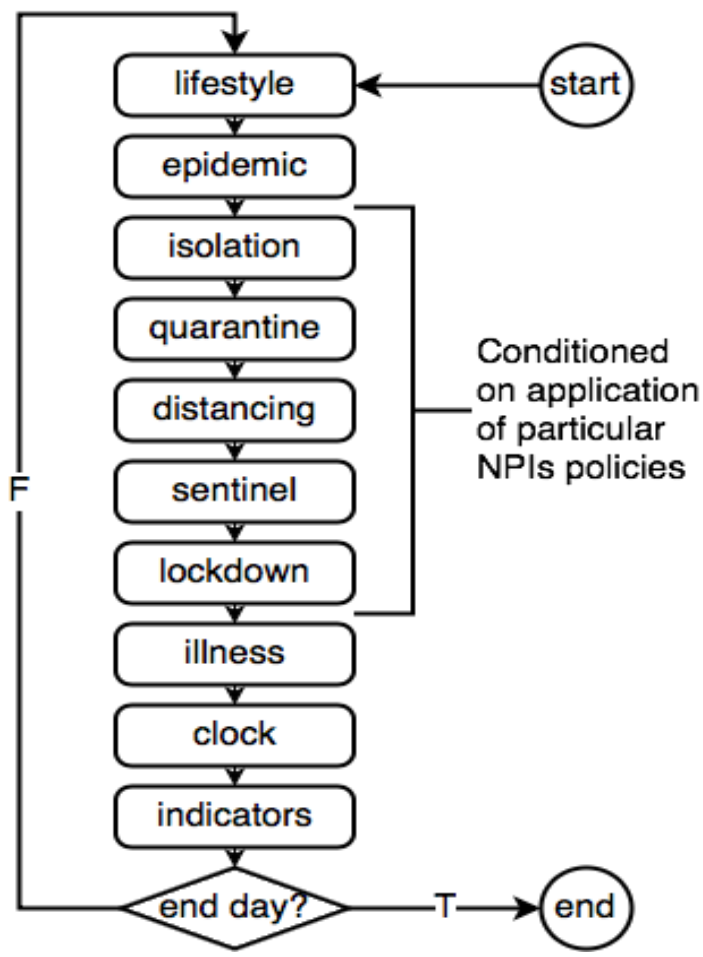

Fig. 2. Diagrama de flujo eventos. Fuente: elaboración propia.

isolation: cuando se aplica el NPI de aislamiento de casos (CI), los casos sintomáticos que aún no se han testado se confinan en sus hogares y se etiquetan como casos confirmados para que tengan que permanecer aislados (su movimiento estará restringido). Si se levanta el CI durante la simulación, los casos confinados se etiquetan de nuevo como no confirmados $\mathrm{y}$, por lo tanto, se dejan en libertad para dejar su aislamiento.

quarantine: cuando se aplica el NPI de cuarentena domiciliaria (HQ), los compañeros de residencia de casos confirmados son enviados a su hogar y su movimiento quedará restringido; permanecerán en cuarentena a menos que se levante HQ durante la simulación.

distancing: cuando el NPI de distancia social (SD) está habilitado, cada agente se mantiene alejado de cualquier otro agente dentro de un radio ligeramente superior a la distancia mínima permitida; para ello cada agente elige al agente más cercano en su vecindad y se orienta en la dirección opuesta. sentinel: cuando se aplica el NPI de Pruebas Centinela (ST), se habilitan ambulancias que se mueven por toda la ciudad realizando pruebas en masa de cualquier agente que se encuentre en su camino. Por lo tanto, cada ambulancia busca a su alrededor agentes que no hayan sido testados anteriormente, realiza la prueba en ellos, marca a estas personas como testadas y, para aquellas que den resultado positivo, las envía a aislamiento etiquetándolas como casos confirmados.

Cada ambulancia se suministra con un inventario de pruebas definidas como un parámetro del modelo (\%-tests); sin embargo, si se quedan sin pruebas durante la simulación, es posible reponerlas con un nuevo suministro de existencias. Resaltamos que este NPI se puede aplicar localmente dentro de cada zona si el parámetro zonal está habilitado. Por último, una vez que se hace la prueba a un agente susceptible, no se volverá a testear en el futuro, a menos que se infecte, en cuyo caso se restablecerá su condición a no testado, como se explicó anteriormente en 
el evento epidemic.

lockdown: cuando se aplica la NPI de Encierro Total (TL), todos los agentes quedan confinados en casa, excepto algunos con permisos de movimiento. Este último está destinado a representar a personas que salen a comprar alimentos o medicinas o trabajadores de servicios esenciales, elegidos al azar cada día acorde con una proporción de la población definida con un parámetro modelo (\%-permits).

Debido a que nuestro modelo considera las divisiones zonales, por lo tanto, es posible levantar la restricción en una o varias zonas mientras se mantiene el resto bloqueado (esto se puede hacer pulsando el botón de zona de desbloqueo en el panel del simulador). Si la TL se desactiva durante la simulación, todos los agentes se desbloquean para que puedan moverse libremente, a menos que, por supuesto, sean casos confirmados. La activación o el levantamiento de TL tienen efecto desde la medianoche del día siguiente a su aplicación.

illness: en esta etapa se simula el progreso de la enfermedad para toda la población de agentes infecciosos, de acuerdo con los eventos de transición del modelo SÎRE $E_{C A R D S}$ descritos anteriormente. La secuencia de aplicación de estos eventos es la siguiente: $\hat{\imath} \rightarrow R, \hat{l} \rightarrow E, \hat{l}+$ Severity, $\hat{l}$ + Deadliness, $\hat{\imath}+$ Hospitalised, y, por último, $\hat{I}+I C U$-admitted.

clock: este evento actualiza los contadores de día y hora que dependen del número de tictacs (o iteraciones) que se necesitan para completar un día simulado, según lo definido por el parámetro del modelo correspondiente.

indcators: aquí se calculan las estadísticas de simulación y los indicadores de epidemia, que incluyen: Historial del número de personas infectadas por día durante la línea de tiempo de la simulación.

Dos estimados del número de reproducción.

El primero utiliza un enfoque a nivel de población que toma datos de incidencia acumulada en un día determinado, conocido como tasa de ataque [16]; aquí R0 se calcula como (1), la inversa de la proporción de susceptibles después del último día terminación de la simulación de la epidemia [17]:

$$
R_{0}=\frac{\ln S_{0}-\ln S_{t}}{S_{0}-S_{t}}
$$

donde $S_{0}$ es la cantidad inicial de susceptibles en el momento de la introducción de los primeros infectados, y $S_{t}$ la cantidad de susceptibles en el momento $t$. El segundo estimado se obtiene a escala individual, calculado directamente mediante rastreo a los agentes, a sus contactos y contagios individualmente a lo largo de la línea de tiempo de la epidemia, y luego tomando el promedio, como se observa en (2):

$$
\bar{R}_{0}=\frac{1}{\left|C^{t}\right|} \sum_{k} C_{k}^{t}
$$

donde $C_{k}^{t}$ es el número de contagios realizados por el agente $k$ hasta el tiempo $t$, y $\left|C^{t}\right|$ es el recuento de agentes que efectivamente han hecho un contagio hasta ese momento. En este sentido, en lugar de hacer suposiciones sobre el valor del número de reproducción, nuestro modelo lo mide como una propiedad emergente de la dinámica de la epidemia. Ambos indicadores, $R_{0}$ y $\bar{R}_{0}$ generalmente siguen tendencias similares, aunque en algunos escenarios pueden diferir ampliamente como se ha reportado previamente [18], tal como como se puede verificar en los gráficos de salida del simulador.

1) El tiempo de duplicación calculado según [19] , como se observa en (3):

$$
D T=\frac{\ln 2}{\ln N_{I}^{t}-\ln N_{I}^{t-1}}
$$

con $N_{I}^{t}$ el número de agentes infectados en el tiempo $t$. 
2) Adicionalmente, se registran $u$ obtienen las siguientes estadísticas: cantidad total de agentes sanos (susceptibles o recuperados), cantidad total de muertes, cantidad total de agentes infectados, cantidad de agentes infectados y confirmados hoy, nuevos casos hoy, número de pruebas positivas y porcentaje de inmunidad de rebaño.

La letalidad se informa como la tasa de fatalidad por infección (IFR) y la tasa de fatalidad por casos confirmados (CFR), que se calculan cada día como el número acumulado de muertes por número acumulado de infecciones totales o casos confirmados, respectivamente.

3) Por último, se obtienen una serie de gráficos que muestran la dinámica de la simulación con respecto al tiempo: gráfico SIRE (Susceptibles, Infectious, Recovered, Extinct), gráfico I+CARDS (Confirmed, Asymptomatic, Risky, Severe, Deadly), gráfico de demanda de camas de Hospital y UCIs, y gráfico de curvas de estimaciones de $R_{0}$ y tiempo de duplicación.

\section{SIMULADOR}

La herramienta de simulación se implementó en el lenguaje NetLogo v.6.1 (13 de mayo de 2019) y está disponible gratuitamente para experimentación en el sitio web de ModelingCommons (ver http://modelingcommons.org/browse/one_m odel/6374). NetLogo es una plataforma de software ampliamente utilizada para modelado ABM, que integra además del lenguaje, un área de vista gráfica y un banco de pruebas para el diseño experimental [20]. El simulador implementa los NPI, el modelo Sî $R E_{C A R D S}$ epidémico y las reglas de comportamiento del agente descritas anteriormente.

En la Fig. 3 se muestra una instantánea del área de vista de simulación. Allí los agentes se representan con diferentes formas según la zona donde residen. El color del agente representa su estado extendido (saludable: mismo color que la zona terrestre; inmune: blanco; enfermo: rojo o amarillo si es asintomático; muerto: X negra). También se pueden ver agentes especiales destinados a implementar algunos de los NPIs, como hogares para cuarentena domiciliaria $\mathrm{y}$ ambulancias para pruebas centinela.

El panel de control está organizado en secciones relacionadas con la configuración general de la ciudad y de la COVID-19, monitores de indicadores epidémicos, parámetros, comandos de acción para ejecutar la simulación y una sección especial para la activación de NPI con sus parámetros correspondientes. En la Fig. 4 se muestra una instantánea del panel.

Además, la herramienta también incluye otro panel con salidas de gráficos que muestran la dinámica de la simulación, como se mencionó en la sección anterior. Un ejemplo de las curvas obtenidas en una ejecución particular del modelo se muestra en la Fig. 5. 
Herramienta basada en agentes para la valoración del impacto de intervenciones no farmacéuticas contra la COVID-19

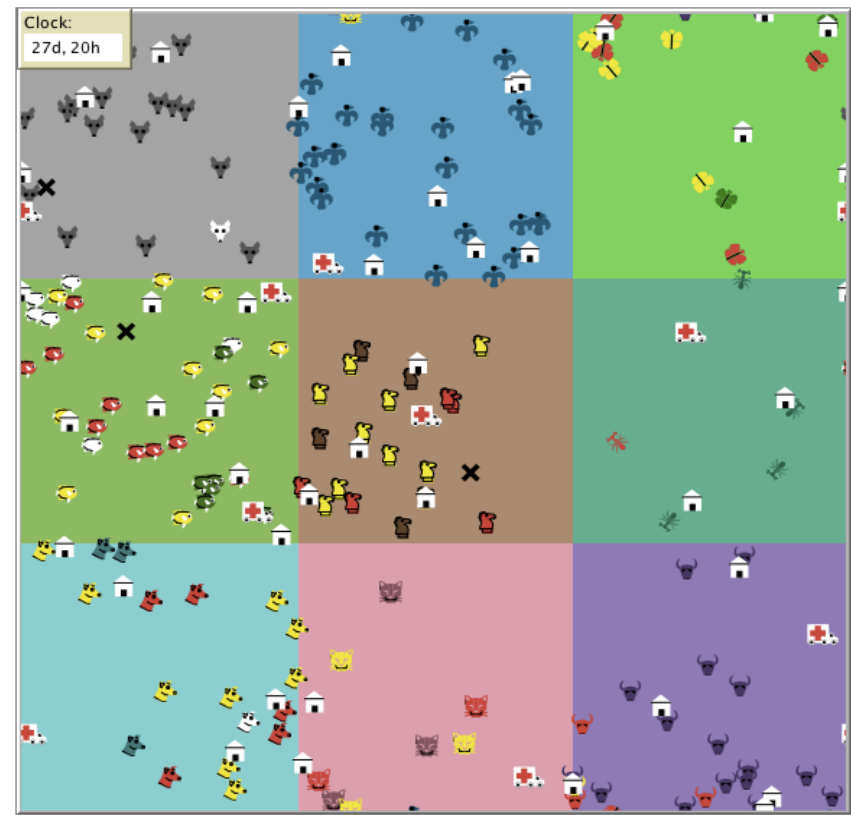

Fig. 3. El área de vista del simulador rowse/one_model/6374 Fuente: elaboración propia.

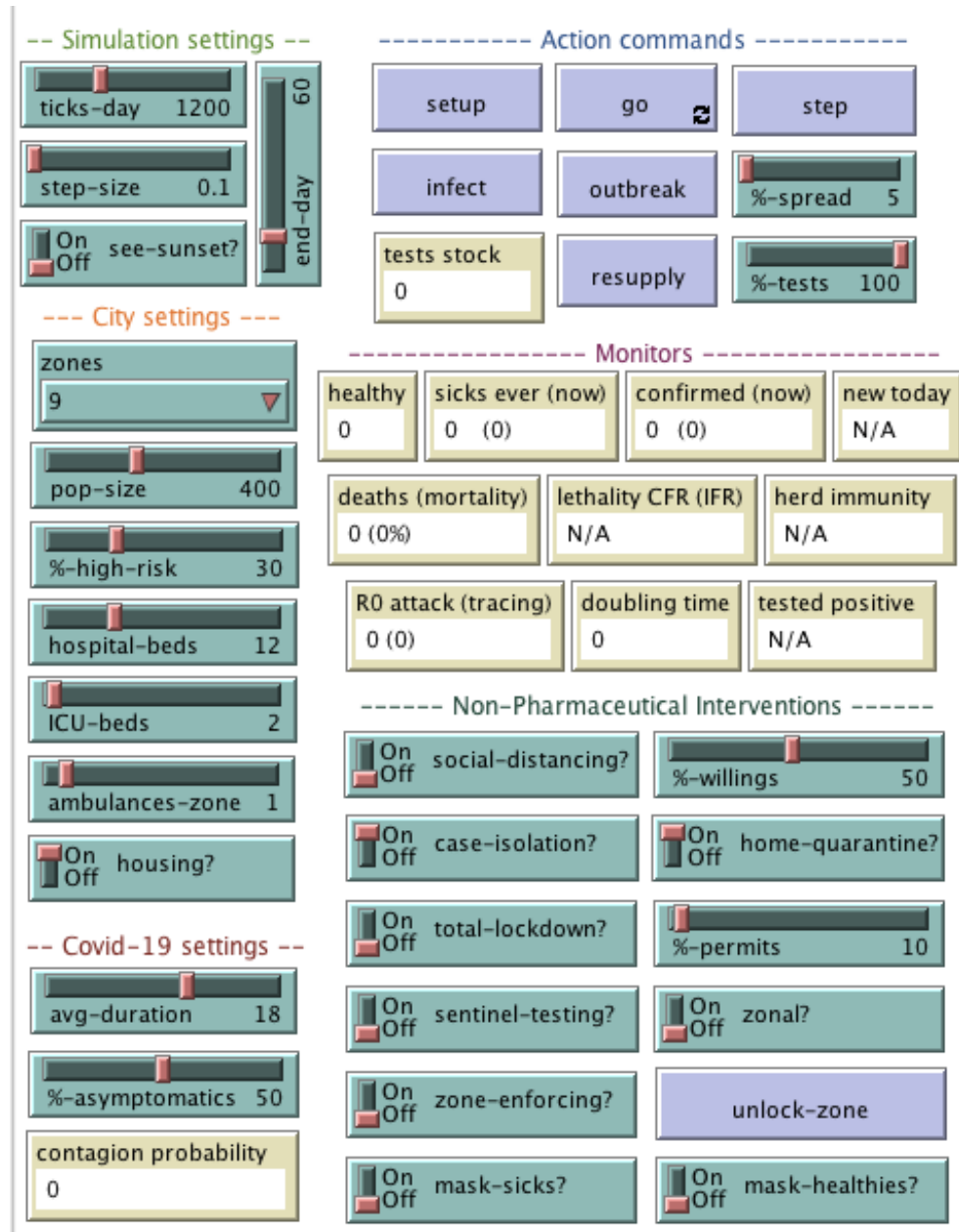

Fig. 4. Panel de control del simulador. Fuente: elaboración propia. 

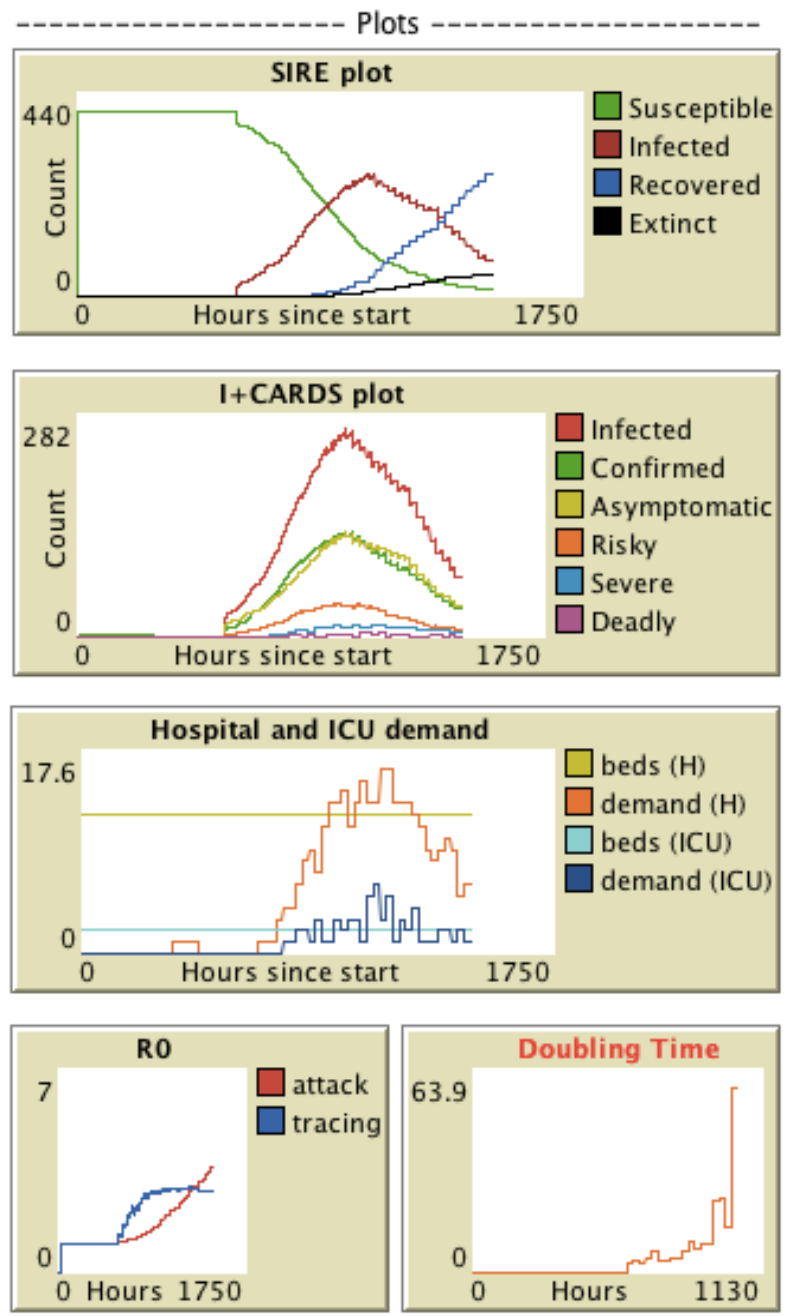

Fig. 5. Área de gráficos de salida del simulador. Fuente: elaboración propia.

\section{ESCENARIOS RESULTADOS \\ HIPOTÉTICOS Y}

En esta sección describimos una serie de escenarios para ilustrar cómo usar el simulador para realizar una evaluación rápida del efecto de los NPI para contener la propagación de la epidemia.

Comenzamos con un escenario de referencia donde no se hace nada para contener la epidemia (Do Nothing, DN).

Luego simulamos escenarios donde se aplican los NPI individuales. Finalmente, simulamos escenarios en los que estos NPI se combinan con la estrategia de restricción zonal para verificar el impacto potencial que este tipo de política puede tener en situaciones de la vida real.
Es importante resaltar que la utilización de la herramienta para la toma de decisiones por parte de las autoridades de salud, requerirá el uso de datos reales para la calibración de los parámetros del modelo.

La descripción de estos escenarios se da en la Tabla 1. Los parámetros generales, de ciudad y COVID-19 utilizados en todas las simulaciones se describen en la Tabla 2. Para cada escenario, se supone que la epidemia comienza con un "paciente cero" sembrado al azar en cualquier zona de la ciudad, a las 0d: $12 \mathrm{~h}$ después del inicio de la simulación. La aplicación de las políticas NPI configuradas comienza a las 04d: $00 \mathrm{~h}$.

Para cada escenario realizamos un experimento que consta de 30 repeticiones 
de ejecución del modelo de simulación con una duración de 60 días. Recopilamos los resultados del recuento de agentes sanos, muertes e índice de inmunidad, así como algunos indicadores epidémicos obtenidos al final del periodo de la simulación.

Los promedios y desviaciones estándar se resumen en las Tablas 3 y 4.

Estos resultados reflejan la efectividad de los diferentes escenarios de NPI. En todos los escenarios, el recuento de muertes varía de 65.1 a 5.3, mientras que la tasa de inmunidad varía de $100 \%$ a $7 \%$.

Se observa en los escenarios individuales de NPI, que las pruebas centinela alcanzan el mayor número de personas sanas $(381 \pm 13$ en promedio), de las cuales aproximadamente el $34 \%$ adquirió inmunidad a la enfermedad, mientras que también logró las tasas de mortalidad más bajas de aproximadamente $4 \%$ (19 \pm 13 muertes); por lo tanto, ST parece ser el NPI individual que obtiene el mayor impacto en contener la epidemia.

Por lo tanto, si las autoridades necesitan priorizar la aplicación de intervenciones, estos resultados sugieren que se debe prestar especial atención a ST.

Ahora bien, con respecto al recuento de supervivientes sanos, la estrategia de mascarillas de protección para todos (MP everyone), logra el segundo mejor resultado (361 \pm 43$)$ seguido por el Encierro Total, TL $(345 \pm 25)$.

Tabla 1. Descripción de escenarios simulados. Fuente: elaboración propia.

\begin{tabular}{|c|c|}
\hline Escenario & Descripción \\
\hline $\mathrm{DN}$ & $\begin{array}{l}\text { Escenario de referencia. El paciente cero se siembra a las 00d: } 12 \mathrm{~h} \text { y la epidemia se } \\
\text { desarrolla sin intervenciones. }\end{array}$ \\
\hline SD & $\begin{array}{l}\text { Escenario de referencia más distanciamiento social activado con el } 50 \% \text { de la población } \\
\text { dispuesta a cumplir (\%-willings). }\end{array}$ \\
\hline $\mathrm{CI}$ & Escenario de referencia más aislamiento de casos, sin cuarentena domiciliaria de familiares. \\
\hline TL & $\begin{array}{l}\text { Escenario de referencia más encierro total con el } 10 \% \text { de las personas con permiso de } \\
\text { abandonar los hogares (\%-permits). }\end{array}$ \\
\hline $\mathrm{ST}$ & $\begin{array}{l}\text { Escenario de referencia más pruebas centinela sin limitación zonal. Se proporciona un stock } \\
\text { suficiente de pruebas a cada ambulancia. }\end{array}$ \\
\hline $\mathrm{ZE}$ & $\begin{array}{l}\text { Escenario de referencia más aplicación de zona o restricción de movilidad dentro de cada } \\
\text { una de las nueve zonas. }\end{array}$ \\
\hline MP & $\begin{array}{l}\text { Escenario de referencia más protección de máscara con tres escenarios: todos usan } \\
\text { máscaras, solo enfermos o solo saludables }\end{array}$ \\
\hline $\mathrm{SD}+\mathrm{ZE}$ & Igual que SD pero con la restricción zonal activada. \\
\hline $\mathrm{CI}+\mathrm{ZE}$ & Igual que CI pero con la restricción zonal activada. \\
\hline $\mathrm{TL}+\mathrm{ZE}$ & Igual que TL pero con la restricción zonal activada. \\
\hline $\mathrm{ST}+\mathrm{ZE}$ & Igual que ST pero con la restricción zonal activada. \\
\hline $\mathrm{MP}+\mathrm{ZE}$ & Igual que MP todos con máscara, más la restricción zonal activada. \\
\hline
\end{tabular}


Herramienta basada en agentes para la valoración del impacto de intervenciones no farmacéuticas contra la COVID-19

Tabla 2. Configuración utilizada en todos los escenarios Fuente: elaboración propia.

\begin{tabular}{llc}
\hline Parámetro & Descripción & Valor \\
\hline pop-size & Número total de personas (agentes) & 400 \\
zones & Cantidad de zonas residenciales & 9 \\
days & Duración de la simulación & $30 \%$ \\
\% high-risk & \% de población con comorbilidades & 12 \\
hospital-beds & Cantidad de camas de hospital disponibles & 2 \\
ICU-beds & Cantidad de camas de UCI disponibles & 1 \\
ambulances-zone & Cantidad de ambulancias centinelas por zona & 18 días \\
avg-duration & Periodo de recuperación promedio & $50 \%$ \\
\% asymptomatic & $\%$ de pacientes que no muestran síntomas o presentan síntomas leves & 5 \\
\hline
\end{tabular}

Tabla 3. Conteo de sobrevivientes, muertes e inmunidad Fuente: elaboración propia.

\begin{tabular}{|c|c|c|c|c|}
\hline Escenario & Sobrevivientes & Muertes & Inmunes & Inmunidad (\%) \\
\hline$\overline{\mathrm{DN}}$ & $334.9 \pm 8.7$ & $65.1 \pm 8.7$ & $334.8 \pm 8.8$ & $1.00 \pm 0.00$ \\
\hline SD & $338.8 \pm 7.6$ & $58.2 \pm 7.3$ & $329.9 \pm 8.2$ & $0.97 \pm 0.01$ \\
\hline CI & $344.0 \pm 20.0$ & $55.9 \pm 20.0$ & $302.7 \pm 102.5$ & $0.90 \pm 0.30$ \\
\hline $\mathrm{TL}$ & $344.6 \pm 25.0$ & $26.8 \pm 14.4$ & $162.1 \pm 82.4$ & $0.47 \pm 0.24$ \\
\hline $\mathrm{ST}$ & $380.8 \pm 13.0$ & $18.8 \pm 12.6$ & $125.4 \pm 77.7$ & $0.34 \pm 0.21$ \\
\hline $\mathrm{ZE}$ & $339.1 \pm 49.2$ & $36.5 \pm 18.2$ & $211.0 \pm 104.8$ & $0.65 \pm 0.33$ \\
\hline MP everyone & $361.2 \pm 43.7$ & $6.3 \pm 9.1$ & $35.0 \pm 45.2$ & $0.11 \pm 0.15$ \\
\hline MP healthy & $334.0 \pm 6.6$ & $66.0 \pm 6.6$ & $334.0 \pm 6.6$ & $1.00 \pm 0.00$ \\
\hline MP sicks & $329.0 \pm 38.1$ & $49.9 \pm 20.9$ & $265.2 \pm 109.2$ & $0.83 \pm 0.33$ \\
\hline $\mathrm{SD}+\mathrm{ZE}$ & $356.1 \pm 29.3$ & $17.9 \pm 8.8$ & $105.9 \pm 48.8$ & $0.31 \pm 0.16$ \\
\hline $\mathrm{CI}+\mathrm{ZE}$ & $375.0 \pm 39.5$ & $10.4 \pm 8.2$ & $64.0 \pm 55.6$ & $0.18 \pm 0.16$ \\
\hline $\mathrm{ST}+\mathrm{ZE}$ & $394.8 \pm 4.6$ & $5.2 \pm 4.6$ & $34.6 \pm 26.4$ & $0.09 \pm 0.07$ \\
\hline $\mathrm{TL}+\mathrm{ZE}$ & $394.0 \pm 3.4$ & $6.0 \pm 3.4$ & $35.3 \pm 19.7$ & $0.09 \pm 0.05$ \\
\hline MP everyone $+\mathrm{ZE}$ & $388.8 \pm 12.6$ & $5.3 \pm 3.9$ & $28.4 \pm 20.4$ & $0.07 \pm 0.05$ \\
\hline
\end{tabular}


Herramienta basada en agentes para la valoración del impacto de intervenciones no farmacéuticas contra la COVID-19

Tabla 4. Indicadores epidémicos para cada uno de los escenarios. Fuente: elaboración propia.

\begin{tabular}{|c|c|c|c|c|}
\hline Escenario & CFR (\%) & IFR (\%) & $\begin{array}{c}\text { R0 } \\
\text { (attack) }\end{array}$ & $\begin{array}{c}\overline{\mathrm{R}} 0 \\
\text { (tracing) }\end{array}$ \\
\hline $\mathrm{DN}$ & $0.69 \pm 0.05$ & $0.16 \pm 0.02$ & $21.94 \pm 4.33$ & $1.97 \pm 0.05$ \\
\hline SD & $0.61 \pm 0.05$ & $0.15 \pm 0.02$ & $3.97 \pm 0.36$ & $1.87 \pm 0.05$ \\
\hline $\mathrm{CI}$ & $0.28 \pm 0.10$ & $0.14 \pm 0.05$ & $10.15 \pm 8.77$ & $2.57 \pm 0.88$ \\
\hline $\mathrm{TL}$ & $0.49 \pm 0.15$ & $0.11 \pm 0.04$ & $1.54 \pm 0.34$ & $2.85 \pm 0.40$ \\
\hline $\mathrm{ST}$ & $0.10 \pm 0.05$ & $0.10 \pm 0.05$ & $1.19 \pm 0.44$ & $1.72 \pm 0.65$ \\
\hline $\mathrm{ZE}$ & $0.56 \pm 0.08$ & $0.13 \pm 0.03$ & $3.55 \pm 5.41$ & $1.94 \pm 0.14$ \\
\hline MP everyone & $0.33 \pm 0.25$ & $0.07 \pm 0.08$ & $0.84 \pm 0.58$ & $1.22 \pm 0.89$ \\
\hline MP healthy & $0.70 \pm 0.04$ & $0.17 \pm 0.02$ & $22.51 \pm 3.12$ & $1.97 \pm 0.05$ \\
\hline MP sicks & $0.65 \pm 0.13$ & $0.21 \pm 0.22$ & $3.22 \pm 1.25$ & $1.65 \pm 0.58$ \\
\hline $\mathrm{SD}+\mathrm{ZE}$ & $0.50 \pm 0.10$ & $0.12 \pm 0.04$ & $1.29 \pm 0.19$ & $1.93 \pm 0.24$ \\
\hline $\mathrm{CI}+\mathrm{ZE}$ & $0.27 \pm 0.23$ & $0.17 \pm 0.23$ & $1.03 \pm 0.44$ & $2.05 \pm 0.94$ \\
\hline $\mathrm{ST}+\mathrm{ZE}$ & $0.12 \pm 0.07$ & $0.12 \pm 0.07$ & $1.06 \pm 0.05$ & $1.82 \pm 0.30$ \\
\hline $\mathrm{TL}+\mathrm{ZE}$ & $0.54 \pm 0.19$ & $0.17 \pm 0.11$ & $1.06 \pm 0.03$ & $2.59 \pm 0.57$ \\
\hline MP everyone +ZE & $0.59 \pm 0.4$ & $0.15 \pm 0.15$ & $0.96 \pm 0.33$ & $1.48 \pm 0.57$ \\
\hline
\end{tabular}

Este es un resultado interesante, teniendo en cuenta que el costo financiero de proporcionar mascarillas a la población puede ser insignificante en comparación con los costos económicos y sociales de mantener largos bloqueos. Por otro lado, también es interesante notar que el distanciamiento social SD, cuando el $50 \%$ de la población está dispuesta a cumplir, tiene un impacto muy bajo (sobrevivientes: $339 \pm 8$, muertes: $58 \pm 7$, inmunidad: $97 \%$ ), solo comparable con el escenario de referencia DN (sobrevivientes: $335 \pm 9$, muertes: $65.1 \pm 8.7$, inmunidad: $100 \%$ ).

Estos resultados sugieren que más de la mitad de la población debería adoptar hábitos de distanciamiento si se espera que la NPI SD produzca algún impacto de mitigación; quizás para ello se deba reforzar la creación de hábitos voluntarios a través de campañas educativas de cobertura extensa. Por otra parte, se observa que el uso de mascarillas en susceptibles (MP healthy) también obtiene cifras similares a DN, es decir, es una NPI de bajo impacto. El MP de las personas enfermas, en cambio, es más efectivo en reducir la tasa de mortalidad.

Con respecto a la inmunidad de rebaño o cantidad de sobrevivientes con inmunización, luego del distanciamiento social o SD, viene el aislamiento de casos o CI (muertes: $56 \pm 20$, inmunidad: $90 \pm 30$ \%) y las mascarillas de protección solo para enfermos, MP sicks (muertes: $50 \pm 21$, inmunidad: $83 \pm 33 \%$ ); luego encierro total o TL (muertes: $27 \pm 14$, inmunidad: $47 \pm 24$ \%) y pruebas centinela o ST (muertes: $19 \pm$ 17, inmunidad: $34 \pm 21 \%)$. Un patrón detectable en estos resultados es la alta variabilidad de los promedios, que puede explicarse por dos razones. En primer 
lugar, un tema común en todos estos NPI es que realizan el aislamiento de los casos tan pronto como se descubren: CI por autoaislamiento de casos sintomáticos, ST por aislamiento inmediato de los resultados asintomáticos positivos y TL por confinamiento obligatorio de ambos, personas sintomáticas o asintomáticas (o inclusive sanas). En segundo lugar, dado que los experimentos se diseñaron de manera que solo un agente infectado (paciente cero) se siembra al azar al comienzo de la simulación, es probable que, en algunas repeticiones, tan pronto como entren en vigencia estos NPI, el paciente cero sea enviado a aislamiento antes de que él o ella pueda transmitir el virus a otros agentes $y$, por lo tanto, los indicadores permanecen inalterados. Para superar esta anomalía, planeamos diseñar diferentes experimentos en los que las epidemias se inician no con una sola infección sino con un pequeño brote (un pequeño porcentaje de la población correspondiente a casos importados) distribuidos al azar en diferentes zonas de la ciudad. De esta manera, esperamos que una mayor experimentación proporcione evidencia más sólida sobre la variabilidad del impacto de estos NPI.

Otro hallazgo interesante es sobre el número $R_{0}$, que tuvo el mejor resultado con mascarillas para todos (MP everyone, $0.84)$, seguido de las pruebas centinela ST (1.19) y luego encierro total TL (1.54).

Por otro lado, el número $\overline{\mathrm{R}} o$ que se calcula directamente al rastrear los contactos de los agentes, obtuvo igualmente el mejor resultado con mascarillas para todos (MP everyone, 1.22), luego las pruebas centinela (1.72), seguida de distancia social SD (1.87).

Lo anterior corrobora nuevamente la observación respecto a la priorización que se le debe dar a las intervenciones ST u MP. Por otra parte, se observa que las dos medidas del número de reproducción difieren, particularmente en el escenario $\mathrm{DN} \quad\left(R_{0}=21.94, \quad \bar{R}_{0}=1.97\right), \quad$ como mencionamos anteriormente [18]. En vista de estos resultados, creemos que el enfoque de rastreo $\bar{R}_{0} o$ provee una estimación más realista en simulaciones ABM.

Por otro lado, encontramos que el NPI de restricción zonal, ZE, exhibe indicadores interesantes que sugieren que puede tener un impacto de mitigación positivo. En comparación con el escenario base $\mathrm{DN}, \mathrm{ZE}$ mejora la tasa de mortalidad (de $65 \pm 9$ a $37 \pm 18$ ), CFR (de $69 \pm 5 \%$ a $56 \pm 8 \%$ ) e IFR (de $16 \pm 2 \%$ a $13 \pm 3 \%$ ). Dado que esta es una de las novedades de nuestro modelo, decidimos entonces experimentar el efecto de combinar cada NPI individual con la restricción ZE.

Curiosamente, nuestros hallazgos indican que tales combinaciones con la intervención $\mathrm{ZE}$, produjeron el impacto de mitigación más efectivo en la epidemia. Por ejemplo, el recuento de sobrevivientes y muertes resulta mejor que cualquier otro escenario: TL + ZE (sobrevivientes: $394 \pm$ 3, muertes: $6 \pm 3$ ), ST + ZE (sobrevivientes: $395 \pm 5$, muertes: $5 \pm 5$ ) y $\mathrm{MP}$ everyone + ZE (sobrevivientes: $389 \pm 13$, muertes: $5 \pm$ 4). SD + ZE logra una tasa de inmunidad del $31 \%$, un indicador interesante de protección de las personas susceptibles contra una segunda ola de propagación del virus, teniendo en cuenta que este escenario supone que solo el $50 \%$ de la población respeta el distanciamiento. Con respecto a la tasa de letalidad (CFR), estos NPI combinados obtuvieron valores que oscilan entre el $12 \%$ y el $59 \%$, mientras que la tasa de letalidad infectada (IFR) obtuvo valores más optimistas entre el 12 $\%$ y el $17 \%$, un resultado que confirma el fenómeno de sobreestimación relativa solo a los casos confirmados oficialmente en lugar de contagios reales [21].

Nuestros resultados sugieren tres recomendaciones relevantes. En general, si se aplicasen únicamente NPIs individuales, las autoridades deberían dar prioridad a las pruebas masivas (ST como lo denotamos en nuestro modelo) para mitigar el impacto de COVID-19, 
proporcionando para este propósito un inventario suficiente de pruebas para las unidades médicas que realizan campañas de pruebas aleatorias. Además, las mascarillas de protección para todos (MP como las denominamos en este estudio), es incluso una estrategia cuyo costo es más favorable para los gobiernos, comparado con la aplicación de encierros totales largos con consecuentes bloqueos económicos.

Finalmente, la propuesta de NPIs con restricción zonal descrita en este estudio parece apropiada para aumentar el impacto de mitigación de la combinación de otros NPI individuales. Aunque este es un hallazgo interesante, la aplicación práctica de dicha restricción zonal puede resultar difícil ya que las personas generalmente residen y trabajan en diferentes distritos de una ciudad. Por lo tanto, la viabilidad de limitar la movilidad de las personas dentro de los distritos dependerá de la planificación urbana y el desarrollo de suficiente infraestructura descentralizada, como centros industriales, residenciales, tecnológicos, comerciales y financieros distribuidos de manera que motiven a las personas a residir y trabajar dentro del mismo distrito. Lo anterior puede que sea inviable de lograr para la actual pandemia de COVID-19, particularmente en ciudades de América Latina donde la planificación urbana por zonas es limitada, pero es una idea interesante para comenzar a explorar como medida de prevención ante futuras nuevas epidemias que ocurran en el mediano plazo.

\section{CONCLUSIONES}

Dada la naturaleza compleja del comportamiento humano y de las infecciones por virus, intentar modelar todos los mecanismos de la epidemia de COVID-19 puede resultar difícil; se necesita hacer supuestos para simplificar la representación de los agentes y sus interacciones. Nuestro objetivo era centrarnos en encontrar emergencias en la dinámica de esta enfermedad, considerando un amplio conjunto de rasgos para los agentes. En la vida real, por supuesto, hay muchas características de interés que pueden afectar el crecimiento de la epidemia. Aquí omitimos estructuras de población detalladas, como edad, género, profesión, estado de salud o grupos familiares, escolares y laborales, transporte, turnos laborales o impactos económicos, que podrían ser importantes para soportar análisis más especializados.

Por ejemplo, en la evaluación del impacto económico de cierta NPI, la definición de la edad del agente puede ser un factor diferencial en la estimación de los costos que acarrea en el sistema de salud.

Claramente, esas propiedades se contemplan como extensiones del modelo que esperamos abordar en trabajos futuros, así como también la inclusión de centros o sitios de conglomeración y de hábitos de inteligencia colectiva, buscando capturar emergencias inherentes a las dinámicas ocultas de interacción entre las NPI, la dispersión de la COVID-19 y el comportamiento social de los agentes.

No obstante, los resultados obtenidos indican que el modelo presentado puede ser una herramienta útil en relación a una evaluación rápida del impacto potencial de la combinación de NPI en diferentes etapas de la epidemia.

Así mismo, la versatilidad que ofrece el modelado con ABM permitiría ampliar el conjunto de NPI con relativa facilidad, modificando el diseño de las propiedades de los agentes, como es el caso del modelado de hábitos personales como lavado de manos y la estrategia de cuarentenas intermitentes de corta duración, que actualmente nos encontramos desarrollando. 
Herramienta basada en agentes para la valoración del impacto de intervenciones no farmacéuticas contra la COVID-19

\section{CONFLICTOS DE INTERÉS}

Los autores declaran no tener ningún conflicto de interés.

\section{REFERENCIAS}

[1] S. Lai et al., "Effect of non-pharmaceutical interventions to contain COVID-19 in China," nature, May. 2020. https://doi.org/10.1038/s41586-020-2293-x

[2] N. M. Ferguson et al., "Report 9: impact of non-pharmaceutical interventions (NPIs) to reduce COVID19 mortality and healthcare demand," Imp. Coll. UK. Pp. 1-20, Mar. 2020. https://doi.org/10.25561/77482

[3] N. M. Ferguson; D. A. T. Cummings; C. Fraser; J. C. Cajka; P. C. Cooley; D. S. Burke, "Strategies for mitigating an influenza pandemic," Nature, vol. 442, no. 7101, pp. 448-452, Apr. 2006. https://doi.org/10.1038/nature04795

[4] D. Fanelli; F. Piazza, "Analysis and forecast of COVID-19 spreading in China, Italy and France," Chaos, Solitons \& Fractals, vol. 134, pp. 109761, May. 2020. https://doi.org/10.1016/j.chaos.2020.109761

[5] N. Wilson; L. T. Barnard; A. Kvalsig; A. Verrall; M. G. Baker; M. Schwehm, "Modelling the potential health impact of the covid-19 pandemic on a hypothetical european country," medRxiv, Mar. 2020. https://doi.org/10.1101/2020.03.20.20039776

[6] M. Kantner; T. Koprucki, "Beyond just" flattening the curve": Optimal control of epidemics with purely non-pharmaceutical interventions," arXiv Prepr. arXiv2004.09471, Jul. 2020. https://arxiv.org/abs/2004.09471

[7] G. Giordano et al., "Modelling the COVID-19 epidemic and implementation of populationwide interventions in Italy," Nat. Med., vol. 26, pp. 855-860 Apr. 2020. https://doi.org/10.1038/s41591-020-0883-7

[8] S. Ansumali; M. K. Prakash, "A Very Flat Peak: Why Standard SEIR Models Miss the Plateau of COVID-19 Infections and How it can be Corrected," medRxiv, May. 2020. https://doi.org/10.1101/2020.04.07.20055772

[9] W. Bock et al., "Mitigation and herd immunity strategy for COVID-19 is likely to fail," medRxiv, May. 2020. https://doi.org/10.1101/2020.03.25.20043109

[10] J. T. Tuomisto; J. Yrjölä; M. Kolehmainen; J. Bonsdorff; J. Pekkanen; T. Tikkanen, "An agent-based epidemic model REINA for COVID-19 to identify destructive policies,"
medRxiv, pp. 1- 29, Apr. 2020. https://doi.org/10.1101/2020.04.09.20047498

[11] J. Gomez; J. Prieto; E. Leon; A. Rodriguez, "INFEKTA: A General Agent-based Model for Transmission of Infectious Diseases: Studying the COVID-19 Propagation in Bogotá-Colombia," medRxiv, pp. 1- 15, Apr. 2020.

https://doi.org/10.1101/2020.04.06.20056119

[12] S. F. Railsback; V. Grimm, Agent-based and individual-based modeling: a practical introduction. Princeton university press, 2011. URL

[13] A. Ahmed; J. Greensmith; U. Aickelin, "Variance in system dynamics and agentbased modelling using the SIR model of infectious disease," Proceedings of the 26th European Conference on Modelling and Simulation (ECMS), Koblenz, Germany, Oct. 2012, pp 9-15. URL

[14] K. Lano; D. Clark, "Direct Semantics of Extended State Machines.," J. Object Technol., vol. 6, no. 9, pp. 35-51, 2007. https://doi.org/10.5381/jot.2007.6.9.a2

[15] M. Granada-Echeverri; A. Molina-Cabrera; P. Granada-Echeverri, "Spatio-temporal Projection of Covid-19 in Pereira," vol. 23, no. 49, Sept. 2020. https://doi.org/10.22430/22565337.1655

[16] T. Obadia; R. Haneef; P.-Y. Boëlle, "The R0 package: a toolbox to estimate reproduction numbers for epidemic outbreaks," BMC Med. Inform. Decis. Mak., vol. 12, no. 1, pp. 147, Dec. 2012. https://doi.org/10.1186/1472-694712-147

[17] K. Dietz, "The estimation of the basic reproduction number for infectious diseases," Stat. Methods Med. Res., vol. 2, no. 1, pp. 2341, Mar. 1993. https://doi.org/10.1177/096228029300200103

[18] R. Breban; R. Vardavas; S. Blower, "Theory versus data: how to calculate R0?," PLoS One, vol. 2, no. 3, Mar. 2007. https://doi.org/10.1371/journal.pone.0000282

[19] S. T. Bakir, "Compound Interest Doubling Time Rule: Extensions and Examples from Antiquities," Commun. Math. Financ., vol. 5, no. 2, pp. 1- 11, Sep. 2016. URL

[20] U. Wilensky; W. Rand, An introduction to agent-based modeling: modeling natural, social, and engineered complex systems with NetLogo. Mit Press, 2015. http://www.jstor.org/stable/j.ctt17kk851

[21] R. Verity et al., "Estimates of the severity of coronavirus disease 2019: a model-based analysis," Lancet Infect. Dis., vol. 20, no. 6, pp. 669- 677, Jun. 2020. https://doi.org/10.1016/S1473-3099(20)30243$\underline{7}$ 


\section{CONTRIBUCIÓN DE LOS AUTORES}

(D) 1 Conceptualización del modelo; diseño, ejecución y validación de experimentos; revisión, edición y aprobación del documento final.

(D) 2 Conceptualización del modelo; diseño e implementación del código computacional; diseño, ejecución y validación de experimentos; preparación del documento borrador original; revisión, edición y aprobación del documento final. 
Herramienta basada en agentes para la valoración del impacto de intervenciones no farmacéuticas contra la COVID-19 